\title{
A SIW Antipodal Vivaldi Array Antenna Design
}

\author{
Ying Suo, ${ }^{1,2}$ Wei Li, ${ }^{1}$ and Jianzhong Chen ${ }^{1}$ \\ ${ }^{1}$ School of Electronics and Information Engineering, Harbin Institute of Technology, Harbin 150001, China \\ ${ }^{2}$ Electronic Science and Technology Postdoctoral Station, Harbin Institute of Technology, Harbin 150001, China \\ Correspondence should be addressed to Ying Suo; suoyingsing@126.com
}

Received 5 November 2015; Revised 25 December 2015; Accepted 28 December 2015

Academic Editor: Wei Liu

Copyright (C) 2016 Ying Suo et al. This is an open access article distributed under the Creative Commons Attribution License, which permits unrestricted use, distribution, and reproduction in any medium, provided the original work is properly cited.

\begin{abstract}
A kind of compact SIW (substrate integrated waveguide) Vivaldi array antenna is proposed and analyzed. The antenna consisted of 4 Vivaldi structure radiation elements fed by an equal power divider with SIW technology. The radiation element is composed of antipodal index gradient microstrip lines on both sides of the substrate. The measured reflection coefficient of the array antenna is less than $-10 \mathrm{~dB}$ from $8.88 \mathrm{GHz}$ to $10.02 \mathrm{GHz}$. The measured gain of the array antenna is $13.3 \mathrm{~dB}$ on $9.5 \mathrm{GHz}$.
\end{abstract}

\section{Introduction}

The Vivaldi antenna has been used in many microwave engineering applications for its simple structure, low cross polarization, and highly directive patterns characteristics, especially in ultra bandwidth applications such as vehicular wireless communication, radar imaging, and through-thewall imaging [1-3]. Many kinds of Vivaldi antennas have been investigated for wideband application $[4,5]$, and some new antipodal Vivaldi antennas have been designed to improve radiation pattern and directivity $[6,7]$.

An equal power divider is necessary for the antenna array feed network for equal amplitude design. The SIW multiway microwave power divider attracts a lot of attentions because of its advantages such as small structural dimension, high integration, and low cost [8-10]. A Vivaldi antenna array fed by a SIW printed on a thick substrate is proposed in [11]. A compact four-element printed Vivaldi array is designed and investigated in [12].

The SIW is able to be fabricated by standard low cost PCB technique using low loss and low cost material substrates [13]. There is often a higher return loss in an array antenna system than a single antenna one as the feeding network for an array antenna, such as T-junction or Y-junction waveguide, will introduce a discontinuity where reflection increases sharply. To reduce the return loss, many approaches have been proposed. Reference [14] focused on the structure of its linear tapered array antennas, compared the reflection coefficients of three different structures of the array antenna, and found a maximum $-10 \mathrm{~dB}$ bandwidth of $0.8 \mathrm{GHz}$ in $X$ band, though the bandwidth is not wide.

To broaden the bandwidth of the array antenna, a Vivaldi array antenna operating in $X$-band is designed and measured in this paper. Return loss is optimized in both antenna and feed network. Vivaldi antenna is used as the array antenna element for enlarging the bandwidth. To reduce the return loss, metal via-holes are added and adjusted in the SIW. The antenna consisted of 4 Vivaldi antenna elements fed by a SIW equal power divider. Each Vivaldi element is composed of antipodal index gradient microstrip lines on both sides of the substrate, and SIW-microstrip transition structure is designed for measurement and connection. Finally this paper achieved an antenna design operating in $X$-band.

\section{4-Way SIW Power Divider Design}

A SIW is composed of two columns of cylinder metal viaholes in the side faces of a dielectric substrate and metal layers on the upper and lower faces. The transmission characteristics of a SIW are almost the same as those of its equivalent metal waveguide. As SIWs are often integrated with coplanar waveguides or microstrip lines, transition structures should be designed for the connection. The transition structure must have a low insertion loss, a wide working band, and a simple geometry to be fabricated. The SIW connected with the microstrip transition is shown in Figure 1. 


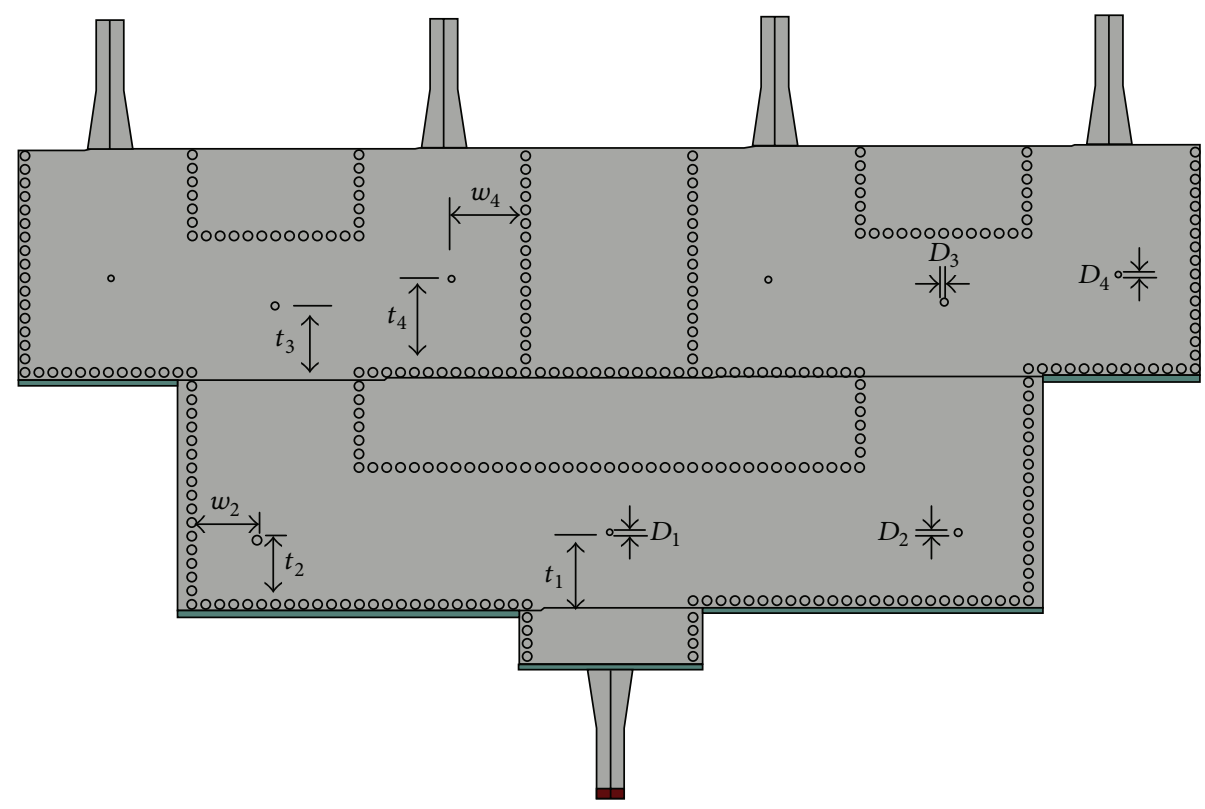

FIGURE 4: 4-way SIW power divider.

and $w_{4}$ determine the position of the via-holes. One via-hole is on the perpendicular bisector of the entire structure and the other two via-holes are on the perpendicular bisectors of the two upper Y-junctions, so there are no parameters $w_{1}$ and $w_{3}$.

To be tested by coaxial cable, five SIW microstrip transitions are connected with the SIW power splitter. The parameters of the microstrip transitions mentioned above are $W, D$, and $H$. $W$ equals 2 and $D$ equals 3.2 , which ensures the impedance transformation of the $50 \Omega$ microstrip line and the SIW. $L$ is optimized to 4.5 for the least reflection loss.

After all the via-holes get their optimized diameter and position that are shown in Table 1, the 4-way SIW power divider is simulated. The permittivity of the substrate is 4.3, and the loss tangent is 0.0015 as used in this design. The simulated $S$-parameters of different ports are shown in Figure 5. It can be seen that the $S_{11}$ parameter is less than $-10 \mathrm{~dB}$ from $8.5 \mathrm{GHz}$ to $10.5 \mathrm{GHz}$. In Figure 5, the transmission coefficient of $S_{21}, S_{31}, S_{41}$, and $S_{51}$ is the same for the equal power division. As shown in Figure 5, the transmission coefficient of the power divider is $-6.3 \mathrm{~dB} \sim$ $-6.6 \mathrm{~dB}$ from $8.9 \mathrm{GHz}$ to $10.07 \mathrm{GHz}$, so it is suitable for the 4 element antenna array design, and the insertion loss is about $0.3 \sim 0.6 \mathrm{~dB}$ from $8.9 \mathrm{GHz}$ to $10.07 \mathrm{GHz}$.

The simulated surface current of the SIW divider on 9.5 GHz is shown in Figure 6. And the simulated electric field of the SIW divider on $9.5 \mathrm{GHz}$ is shown in Figure 7.

\section{Antipodal Vivaldi Antenna Array Design}

The antipodal Vivaldi radiation element is composed of complementary structures on both sides of the substrate. The antenna element is shown in Figure 8. The upper part is the antenna radiator, the middle part is the connection

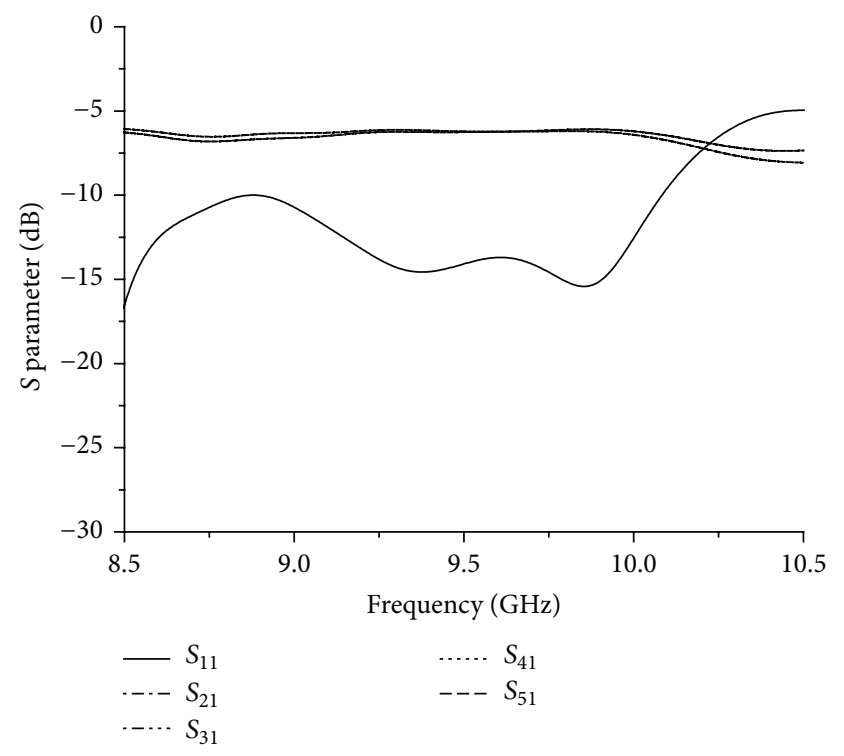

FIGURE 5: $S$ parameters of the 4-way SIW power divider.

structure, and the lower part is the microstrip transition. For the upper radiation part of the antenna shown in Figure 8(a), the radiator is bordered by part of an ellipse curve on the right and an index gradient curve on the left. The index gradient curve whose exponential rate $r$ equals 1 satisfies the following formula:

$$
y=c_{1} \times \mathrm{e}^{r z}+c_{2}
$$

The Vivaldi antenna is directly fed by a microstrip line. Impedance of the microstrip is equal to that of each export of the equal power divider. A SIW-microstrip transition 
TABLE 2: Parameters of the Vivaldi antenna element.

\begin{tabular}{ccccccc}
\hline \multicolumn{7}{c}{ Parameters (mm) } \\
$w_{1}$ & $R$ & $L$ & $L_{1}$ & $L_{2}$ & $L_{3}$ & $w_{s}$ \\
\hline 8.35 & 6.2 & 30.37 & 8.2 & 17 & 12.67 & 11.4 \\
\hline
\end{tabular}

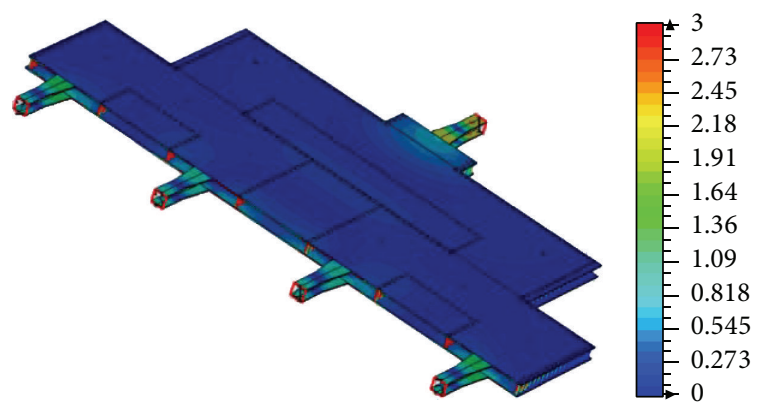

(a) Phase is 0 deg

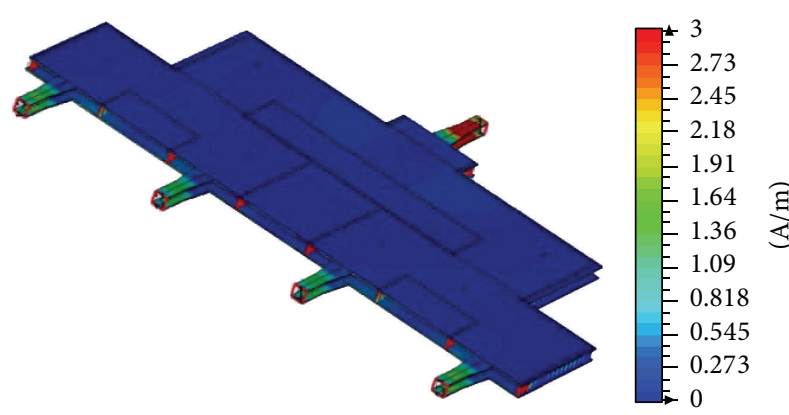

(b) Phase is $90 \mathrm{deg}$

FIGURE 6: Surface current of the 4-way SIW power divider.

structure is designed for measurement and connection. The transition section is a tapered microstrip line.

As is shown in Figure 8, $w_{s}$ is the antenna width, $L$ is the antenna length, $L_{2}$ is set for the length of straight part in SIWmicrostrip transition, $L_{1}$ is the length of the connection part, and $L_{3}$ is the length of the radiator. $R$ is length of the truncated elliptic curve. The parameters of the Vivaldi radiation element are shown in Table 2.

Parameters on Table 2 are also optimized to get the best radiation characteristics of the antenna. The simulation model of antipodal Vivaldi antenna based on 4-way SIW power divider is shown in Figure 9. The dielectric substrate has a relative permittivity of 4.5 and a thickness of $1.6 \mathrm{~mm}$. Parameters of the antenna and the SIW equal power divider equal those in Tables 1 and 2.

To get the optimized parameters of the diameter and position of a via-hole, $S_{11}$ of the 4-way SIW power divider is simulated when one of the parameters of the via-hole varies. Because of the symmetry of the SIW, 4 of the 9 via-holes need to be analyzed.

Figure 10 shows the simulated $S_{11}$ when the diameter of via-hole $1 D_{1}$ varies from 0.3 to 0.6 . It can be seen that the $S_{11}$ curve moves up when $D_{1}$ increase, but not very much. Then 0.5 is picked as the optimized value of $D_{1}$ based on the $S_{11}$ curves.

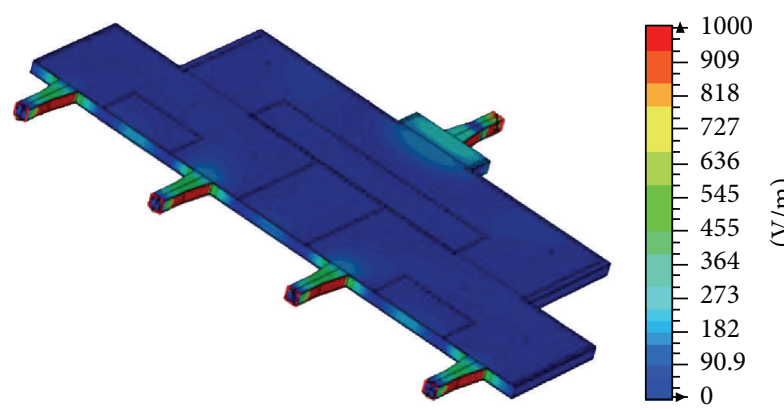

(a) Phase is 0 deg

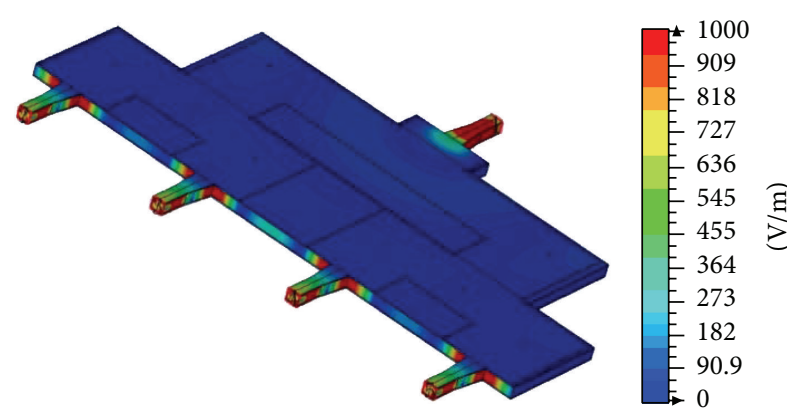

(b) Phase is $90 \mathrm{deg}$

FIgURE 7: Electric field of the 4-way SIW power divider.

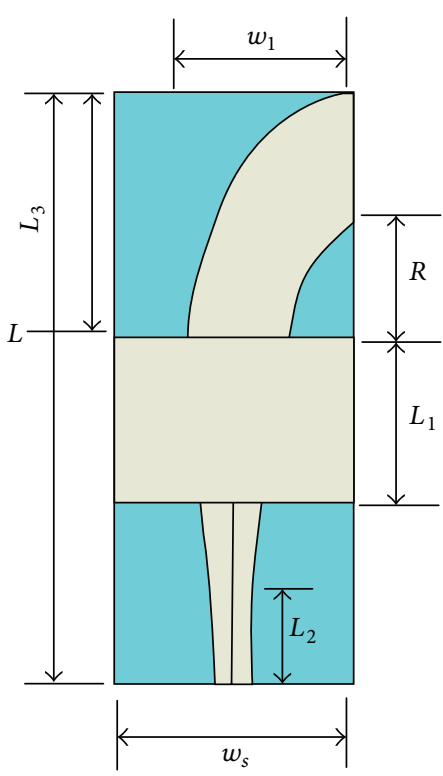

(a) Upper part

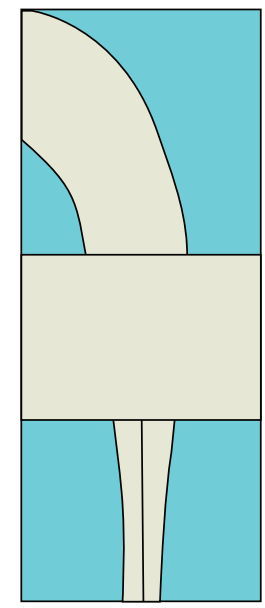

(b) Lower part
Figure 8: Antipodal Vivaldi antenna element.

$S_{11}$ when $t_{1}$ varies from 3 to 6 is shown in Figure 11. In Figure 11, the $S_{11}$ curve firstly moves down and then moves up as $t_{1}$ increases. Therefore the optimized $t_{1}$ lies between 4 and 5. Obviously $S_{11}$ is vastly dependent on $t_{1}$, so repeated simulations were carried out for the least reflection, which have ultimately shown that 4.9 is the best $t_{1}$ value.

Figure 12 has shown the simulated $S_{11}$ curves when the diameter of via-hole $2 D_{2}$ varies from 0.2 to 0.6 . The $S_{11}$ is 


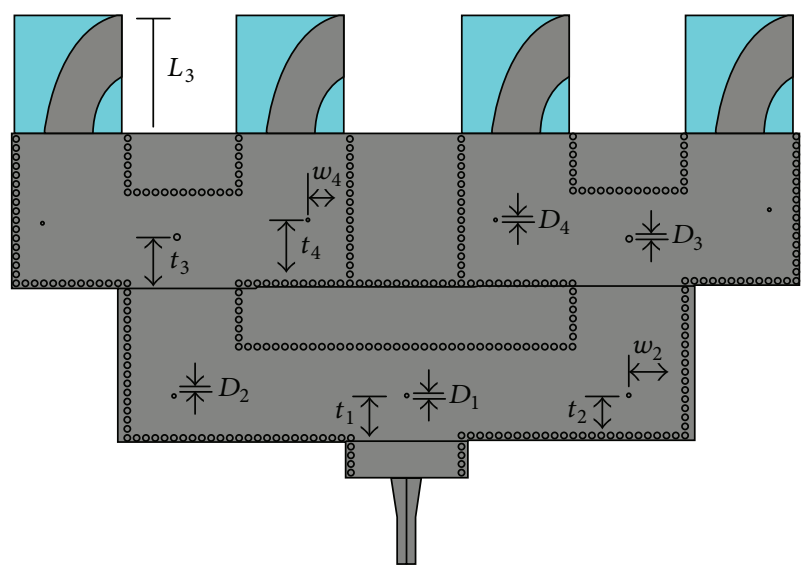

(a) Upper part

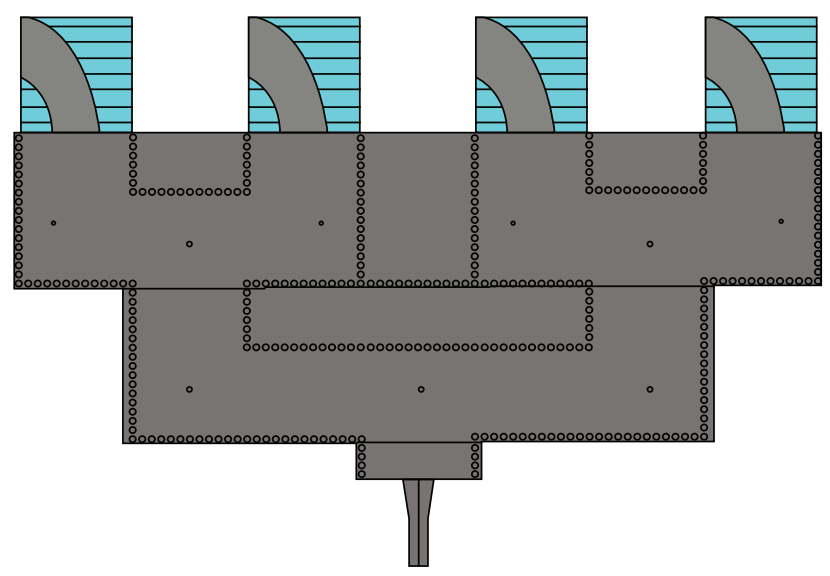

(b) Lower part

FIGURE 9: Simulation model of the SIW Vivaldi antenna array.

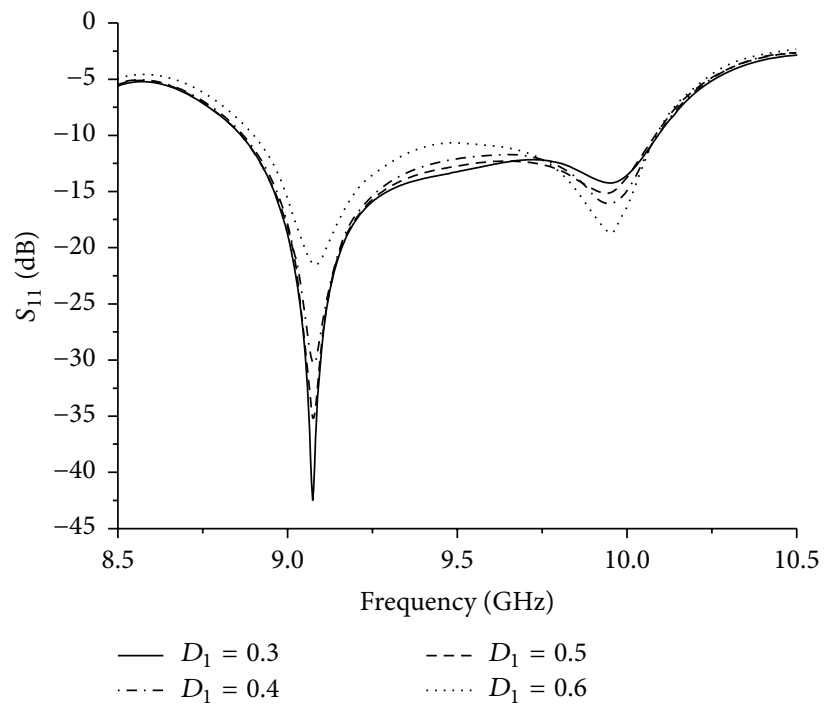

FIgURE 10: $S_{11}$ when $D_{1}$ varies.

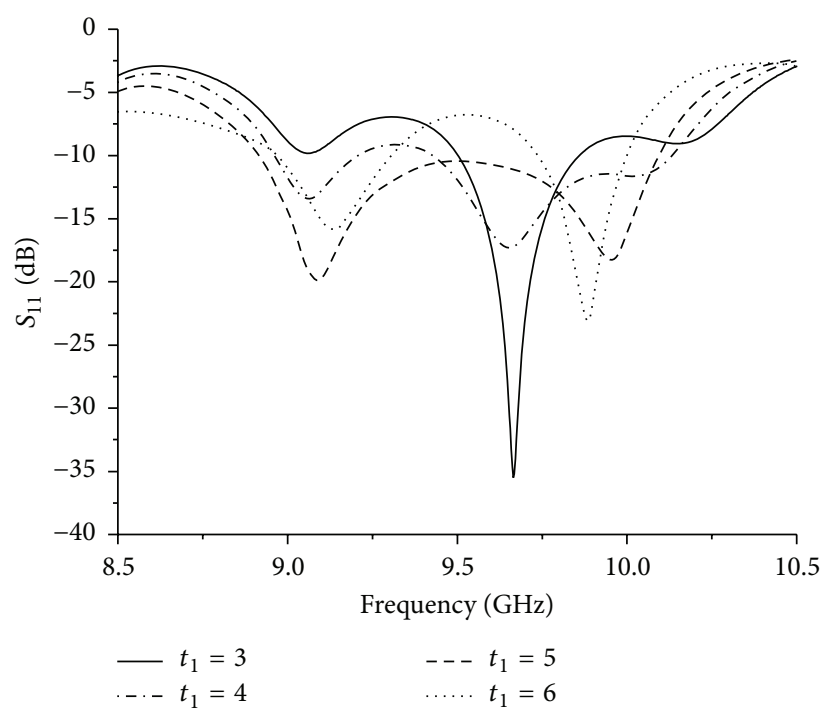

FIGURE 11: $S_{11}$ when $t_{1}$ varies. 


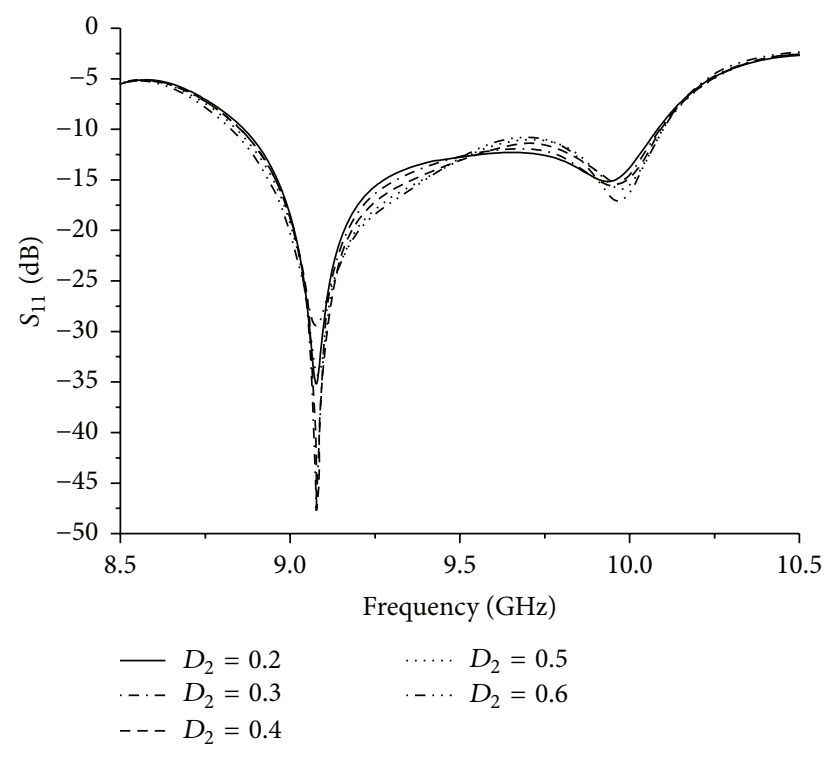

FIGURE 12: $S_{11}$ when $D_{2}$ varies.

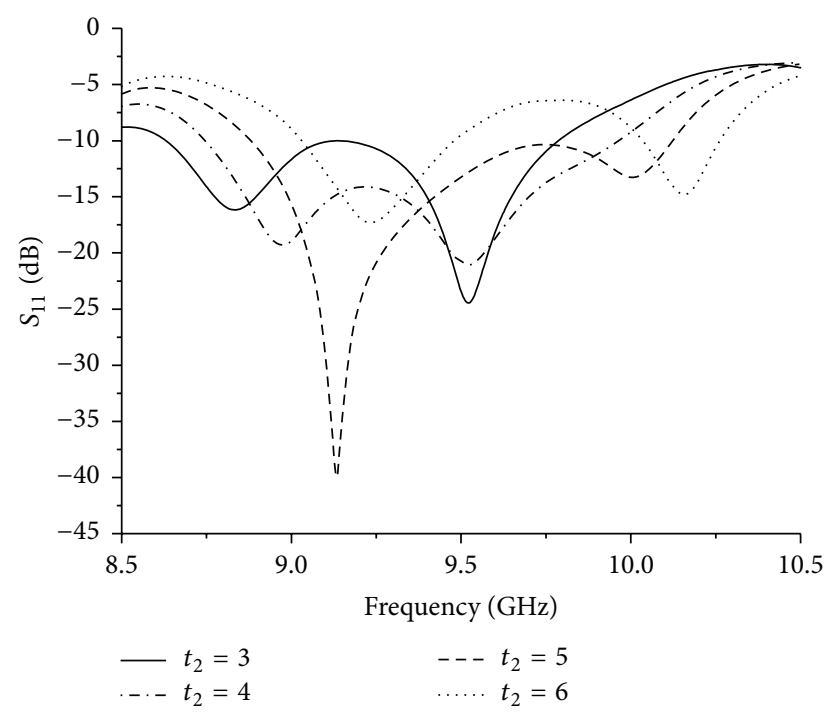

FIGURE 13: $S_{11}$ when $t_{2}$ varies.

also not very dependent on $D_{2}$. For both low reflection loss and wide bandwidth, 0.5 is picked as $D_{2}$.

$S_{11}$ when $t_{2}$ varies from 3 to 6 is shown in Figure 13. When $t_{2}$ increases, the $S_{11}$ curve firstly moves down and then moves up, but the two resonate frequencies of the $S_{11}$ curve moves right continuously. In consideration of both bandwidth and center frequency, $t_{2}=5$ is chosen.

Simulated $S_{11}$ is shown in Figure 14 when $w_{2}$ varies from 3 to 6 . As $w_{2}$ increases, the two center frequencies are moving left. The optimized $w_{2}$ lies between 4 and 6 . After repeated simulation, 5.5 is found to be the optimized $w_{2}$.

$S_{11}$ when $D_{3}$ varies from 0.3 to 0.6 is shown in Figure 15 . The $S_{11}$ curve is much more dependent on $D_{3}$ than $D_{1}$ and

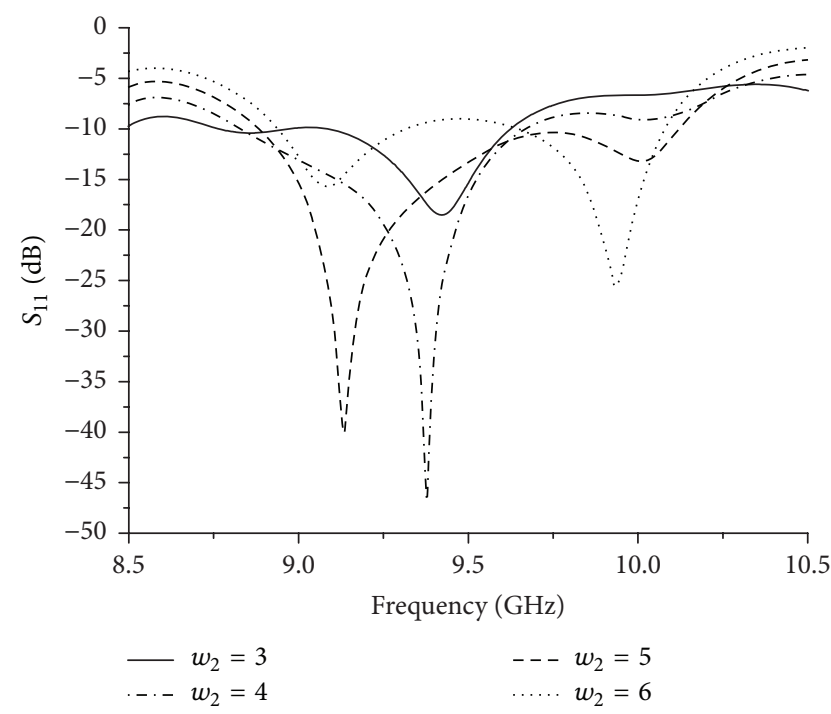

Figure 14: $S_{11}$ when $w_{2}$ varies.

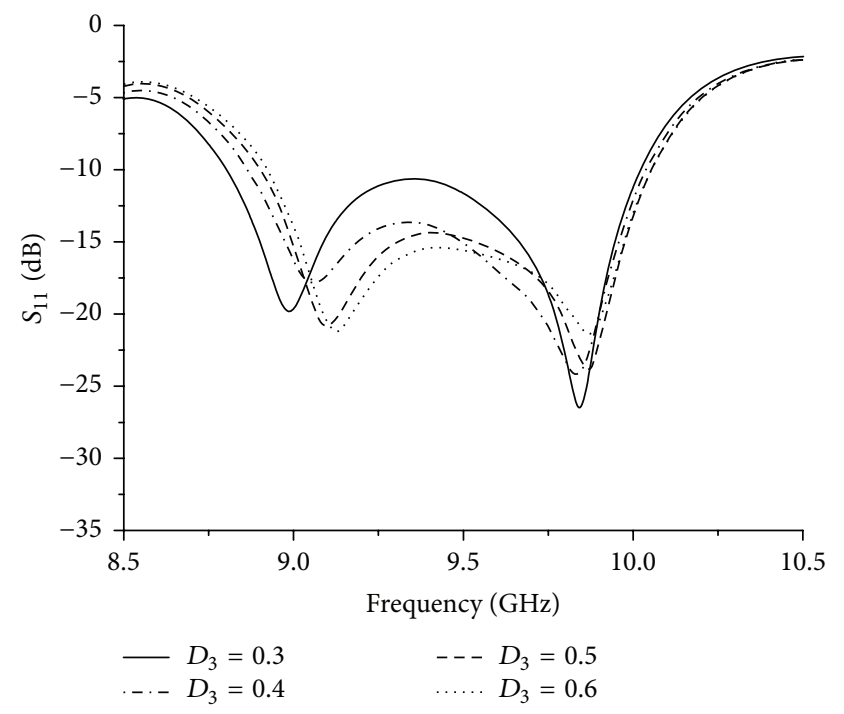

FIgURE 15: $S_{11}$ when $D_{3}$ varies.

$D_{2}$, which moves down as $D_{3}$ increases. 0.5 is picked as the optimized $D_{3}$.

$S_{11}$ when $t_{3}$ varies from 3 to 6 is shown in Figure 16. The $S_{11}$ curve firstly moves down and then moves up, but the two center frequencies keep moving right, as $t_{3}$ increases. 4 is picked as the value of $t_{3}$.

Figure 17 shows the $S_{11}$ curves of the SIW when the diameter of via-hole 4 varies from 0.3 to 0.6 . The high frequency part of the $S_{11}$ curve moves up while the low frequency part moves down as $D_{4}$ increases. So 0.3 is picked as the optimized $D_{4}$ for wider bandwidth.

$S_{11}$ when $t_{4}$ varies is shown in Figure 18. The $S_{11}$ curve firstly moves down and then moves up as $t_{4}$ increases. The optimized $t_{4}$ lies between 7 and 8. Later simulations have found that 7 is the optimized $t_{4}$. 


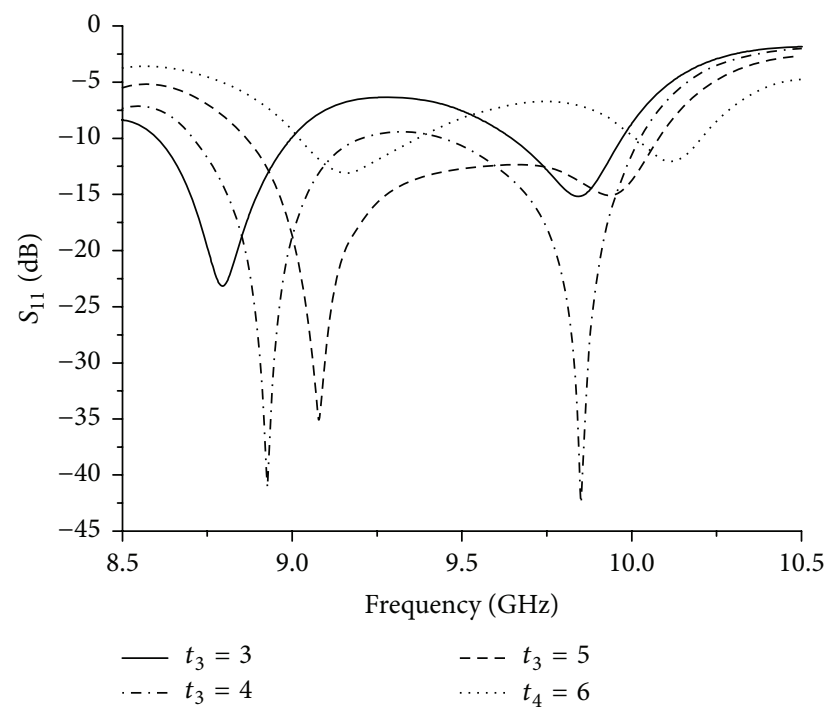

FIGURE 16: $S_{11}$ when $t_{3}$ varies.

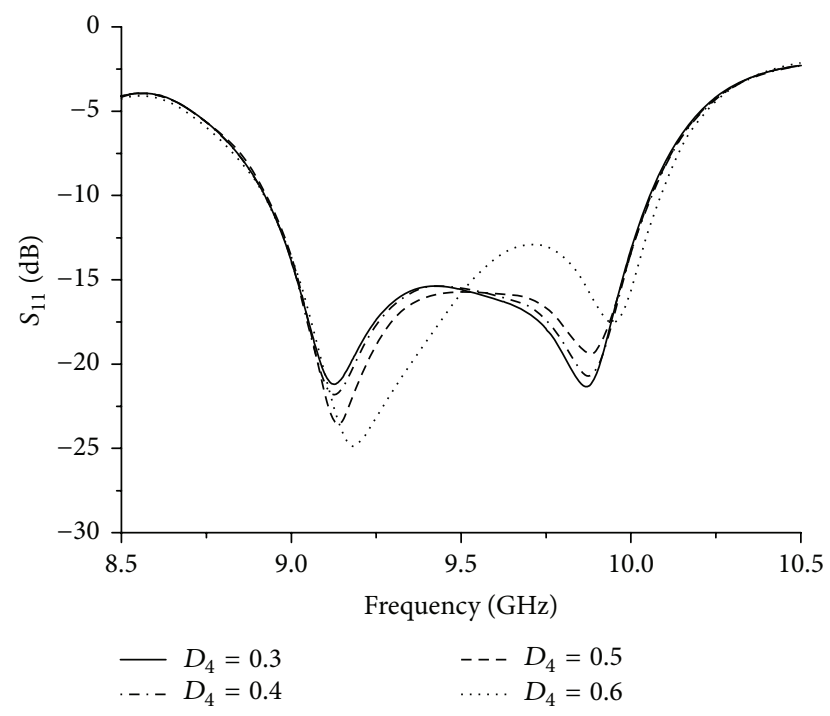

FIGURE 17: $S_{11}$ when $D_{4}$ varies.

$S_{11}$ when $w_{4}$ varies from 3 to 6 is simulated and shown in Figure 19. The bandwidth of the curves changes a little while the center frequencies change more when $w_{4}$ increases. So 4 is picked as the value of $w_{4}$ in consideration of the two center frequencies.

As is shown in Figure 20, a 4-element antipodal SIW Vivaldi antenna array is fabricated and measured. The total dimension of the antenna array is $85 \mathrm{~mm} \times 7 \mathrm{~mm}$ with the feeding cable head. The parameters of the antenna array are $D_{1}=0.5 \mathrm{~mm}, t_{1}=4.9 \mathrm{~mm}, D_{2}=0.5 \mathrm{~mm}, t_{2}=5 \mathrm{~mm}$, $w_{2}=5.5 \mathrm{~mm}, D_{3}=0.5 \mathrm{~mm}, t_{3}=4 \mathrm{~mm}, D_{4}=0.3 \mathrm{~mm}$, and $t_{4}=4 \mathrm{~mm}$.

Figure 21 shows the simulated and measured reflection coefficient. Simulated results show that the reflection coefficient is less than $-10 \mathrm{~dB}$ from $8.9 \mathrm{GHz}$ to $10.07 \mathrm{GHz}$. And the measured reflection coefficient is less than $-10 \mathrm{~dB}$ from

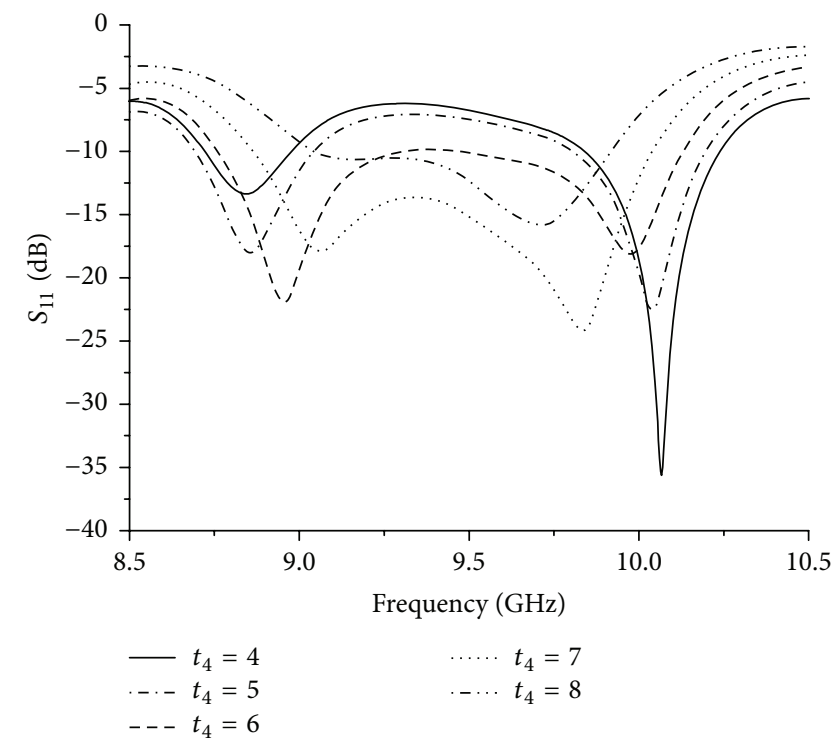

Figure 18: $S_{11}$ when $t_{4}$ varies.

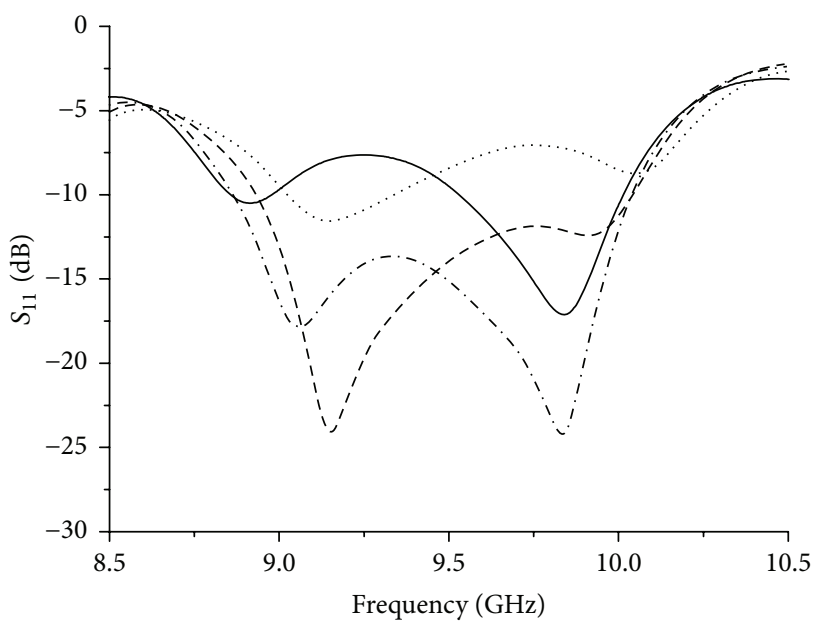

$$
\begin{array}{rlr}
-w_{4}=3 & ---w_{4}=5 \\
\cdots-w_{4}=4 & \cdots \cdots & w_{4}=6
\end{array}
$$

FIGURE 19: $S_{11}$ when $w_{4}$ varies.

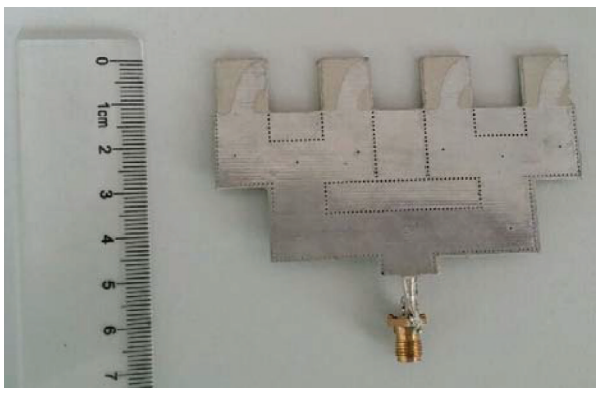

FIGURE 20: Fabricated antipodal SIW Vivaldi antenna array. 


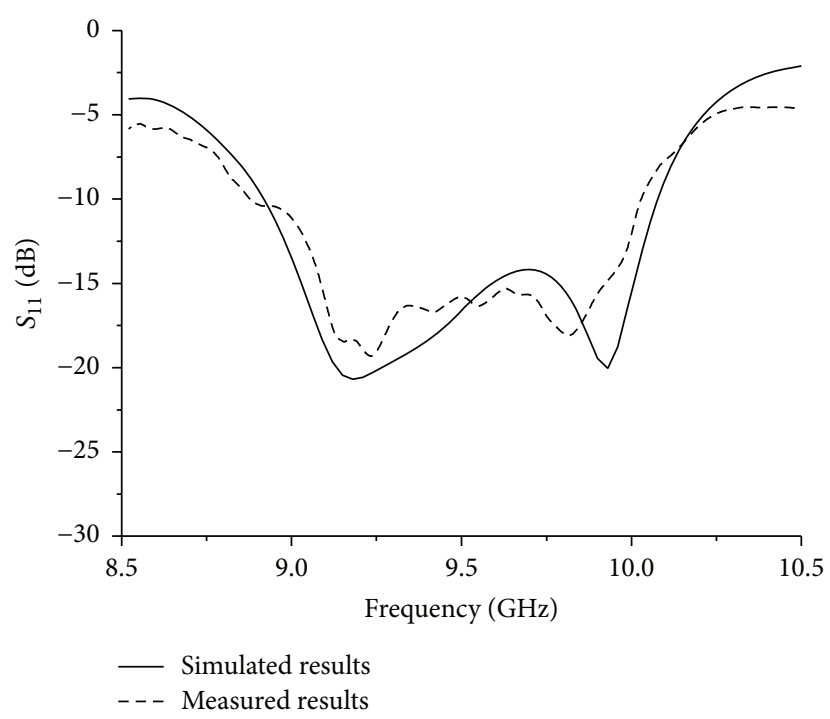

FIGURE 21: Reflection coefficient of the SIW Vivaldi antenna array.

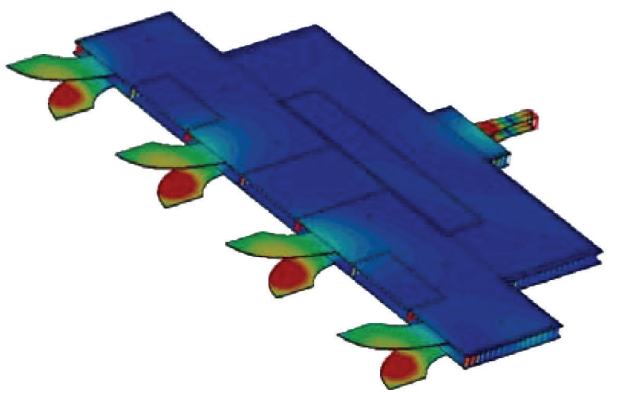

(a) Phase is 0 deg

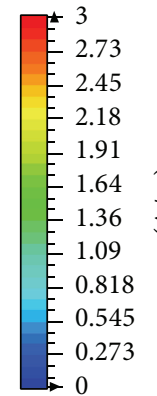

0

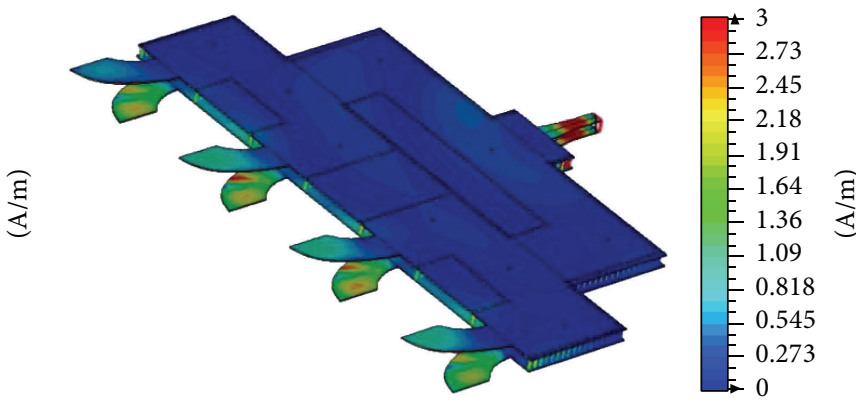

(b) Phase is $90 \mathrm{deg}$

FIGURE 22: Surface current of the SIW Vivaldi antenna array.

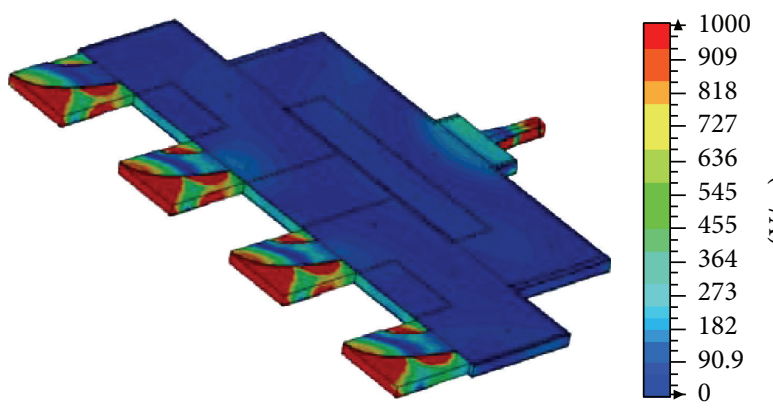

(a) Phase is 0 deg

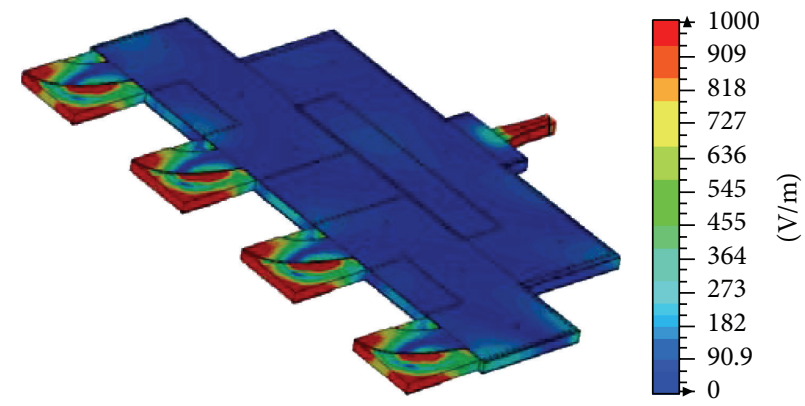

(b) Phase is $90 \mathrm{deg}$

FIGURE 23: Electric field of the SIW Vivaldi antenna array.

$8.88 \mathrm{GHz}$ to $10.02 \mathrm{GHz}$. The measurement shows agreement with the simulation.

The simulated surface current of the SIW Vivaldi antenna array on $9.5 \mathrm{GHz}$ is shown in Figure 22. And the simulated electric field of the SIW Vivaldi antenna array on $9.5 \mathrm{GHz}$ is shown in Figure 23.
The simulated and measured radiation patterns on $9.5 \mathrm{GHz}$ of the SIW Vivaldi antenna array are shown in Figure 24. The array gain can reach $14 \mathrm{~dB}$ on $9.5 \mathrm{GHz}$ in simulation, and the main lobe width is about $17.3^{\circ}$. The measured gain is about $13.3 \mathrm{~dB}$, and the main lobe width is about $16.9^{\circ}$. 


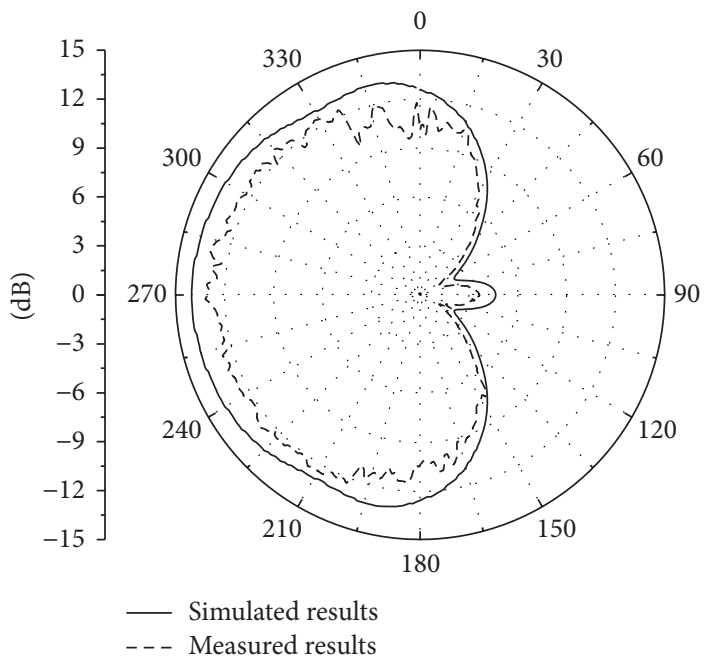

(a) E-plane

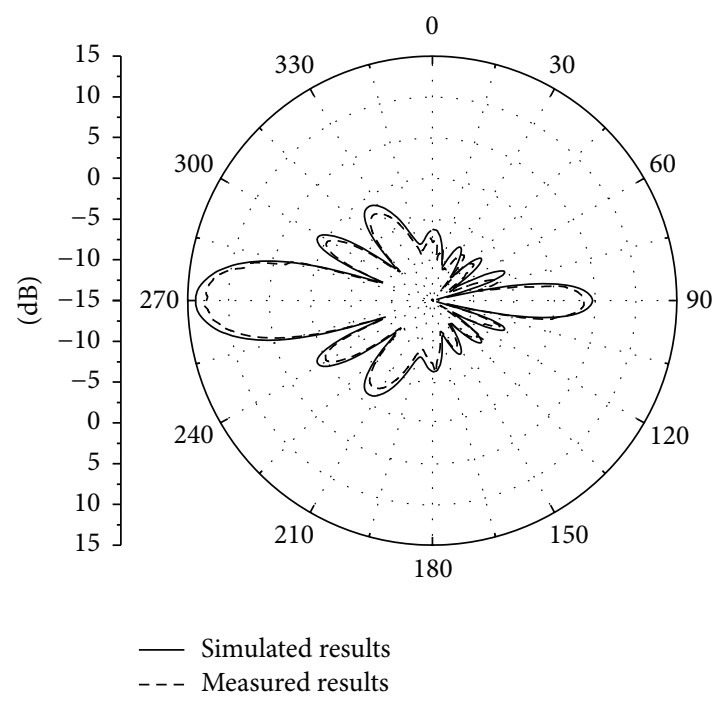

(b) H-plane

FIGURE 24: Pattern of the SIW Vivaldi antenna array on $9.5 \mathrm{GHz}$.

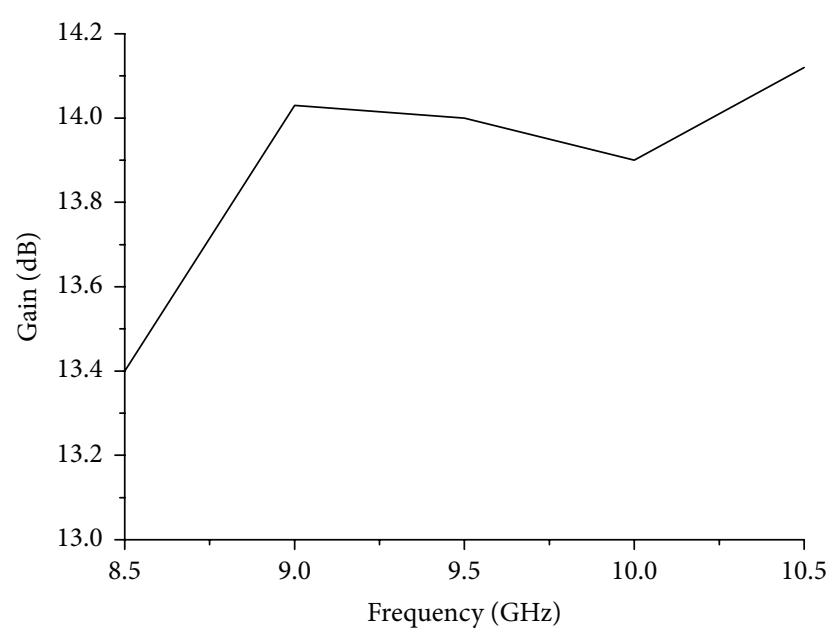

FIGURE 25: Simulated gain of the SIW Vivaldi antenna array.

Figure 25 is the simulated gain in operating band. It is shown that the gain is increased with frequency increasing.

\section{Conclusion}

A 4-way SIW equal power divider is designed, and the impedance characteristics and transmission characteristics satisfy the array design. The Vivaldi radiation element consisted of the antipodal structure. The operating bandwidth of the SIW Vivaldi array antenna is more than $1.1 \mathrm{GHz}$ in $X$ band. And the gain of the array antenna is $13.3 \mathrm{~dB}$ on $9.5 \mathrm{GHz}$.

\section{Conflict of Interests}

The authors declare that there is no conflict of interests regarding the publication of this paper.

\section{Acknowledgment}

The authors would like to express their sincere gratitude to funds supported by "the National Natural Science Funds" (Grant no. 61501145).

\section{References}

[1] S. H. He, W. Shan, C. Fan, Z. C. Mo, F. H. Yang, and J. H. Chen, "An improved vivaldi antenna for vehicular wireless communication systems," IEEE Antennas and Wireless Propagation Letters, vol. 13, pp. 1505-1508, 2014.

[2] X. Zhuge and A. G. Yarovoy, "A sparse aperture MIMO-SARbased UWB imaging system for concealed weapon detection," IEEE Transactions on Geoscience and Remote Sensing, vol. 49, no. 1, pp. 509-518, 2011.

[3] F. Fioranelli, S. Salous, I. Ndip, and X. Raimundo, "Throughthe-wall detection with gated FMCW signals using optimized patch-like and Vivaldi antennas," IEEE Transactions on Antennas and Propagation, vol. 63, no. 3, pp. 1106-1117, 2015.

[4] J. D. S. Langley, P. S. Hall, and P. N. Newham, "Novel ultrawidebandwidth Vivaldi antenna with low crosspolarisation," Electronics Letters, vol. 29, no. 23, pp. 2004-2005, 1993.

[5] R. Natarajan, J. V. George, M. Kanagasabai, and A. Kumar Shrivastav, "A compact antipodal Vivaldi antenna for UWB applications," IEEE Antennas and Wireless Propagation Letters, vol. 14, pp. 1557-1560, 2015.

[6] G. Teni, N. Zhang, J. Qiu, and P. Zhang, "Research on a novel miniaturized antipodal vivaldi antenna with improved radiation," IEEE Antennas and Wireless Propagation Letters, vol. 12, pp. 417-420, 2013.

[7] A. M. De Oliveira, M. B. Perotoni, S. T. Kofuji, and J. F. Justo, "A palm tree antipodal vivaldi antenna with exponential slot edge for improved radiation pattern," IEEE Antennas and Wireless Propagation Letters, vol. 14, pp. 1334-1337, 2015.

[8] S. Germain, D. Deslandes, and K. Wu, "Development of substrate integrated waveguide power dividers," in Proceedings of the IEEE Canadian Conference on Electrical and Computer 
Engineering (CCECE '03), vol. 3, pp. 1921-1924, Montreal, Canada, May 2003.

[9] Z. Hao, W. Hong, H. Li, H. Zhang, and K. Wu, "Multiway broadband substrate integrated waveguide (SIW) power divider," in Proceedings of the IEEE Antennas and Propagation Society International Symposium, pp. 639-642, IEEE, July 2005.

[10] X. Zou, C.-M. Tong, and D.-W. Yu, "Y-junction power divider based on substrate integrated waveguide," Electronics Letters, vol. 47, no. 25, pp. 1375-1376, 2011.

[11] S. Yang, A. Elsherbini, S. Lin, A. E. Fathy, A. Kamel, and H. Elhennawy, "A highly efficient Vivaldi antenna array design on thick substrate and fed by SIW structure with integrated GCPW feed," in Proceedings of the IEEE Antennas and Propagation Society International Symposium, pp. 1985-1988, IEEE, Honolulu, Hawaii, USA, June 2007.

[12] L. Bin, D. Liang, and Z. Jiao-Cheng, "The research of broadband millimeter-wave Vivaldi array antenna using SIW technique," in Proceedings of the International Conference on Microwave and Millimeter Wave Technology (ICMMT '10), pp. 997-1000, IEEE, Chengdu, China, May 2010.

[13] W. M. Abdel Wahab, D. Busuioc, and S. Safavi-Naeini, "Low cost planar waveguide technology-based dielectric resonator antenna (DRA) for millimeter-wave applications: analysis, design, and fabrication," IEEE Transactions on Antennas and Propagation, vol. 58, no. 8, pp. 2499-2507, 2010.

[14] L. Wang, X. Yin, and H. Zhao, "A planar feeding technology using phase-and-amplitude-corrected SIW horn and its application," IEEE Antennas and Wireless Propagation Letters, vol. 14, pp. 147-150, 2015. 


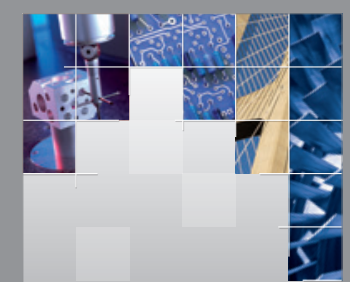

\section{Enfincering}
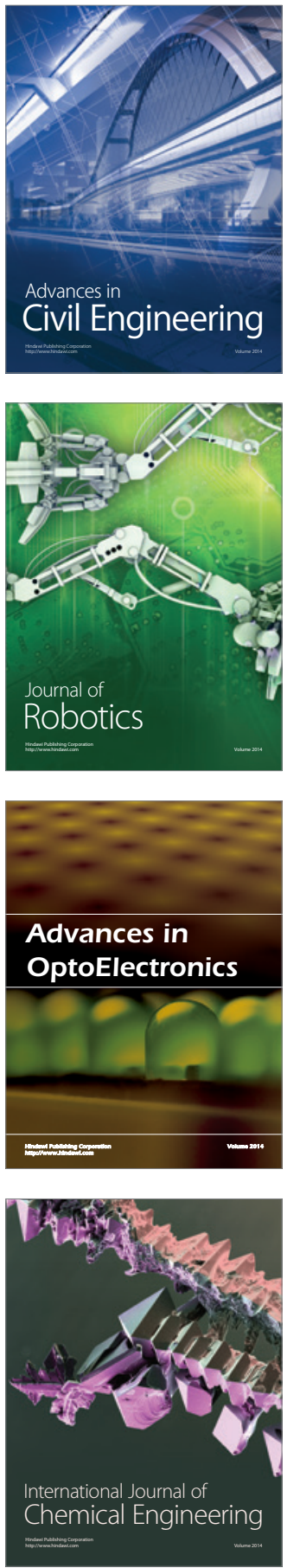

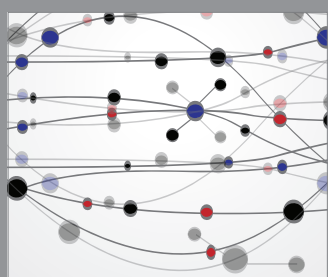

The Scientific World Journal

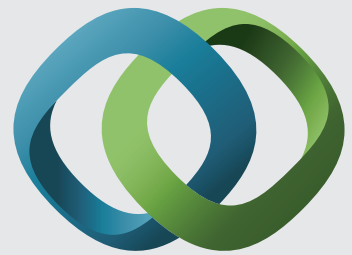

\section{Hindawi}

Submit your manuscripts at

http://www.hindawi.com
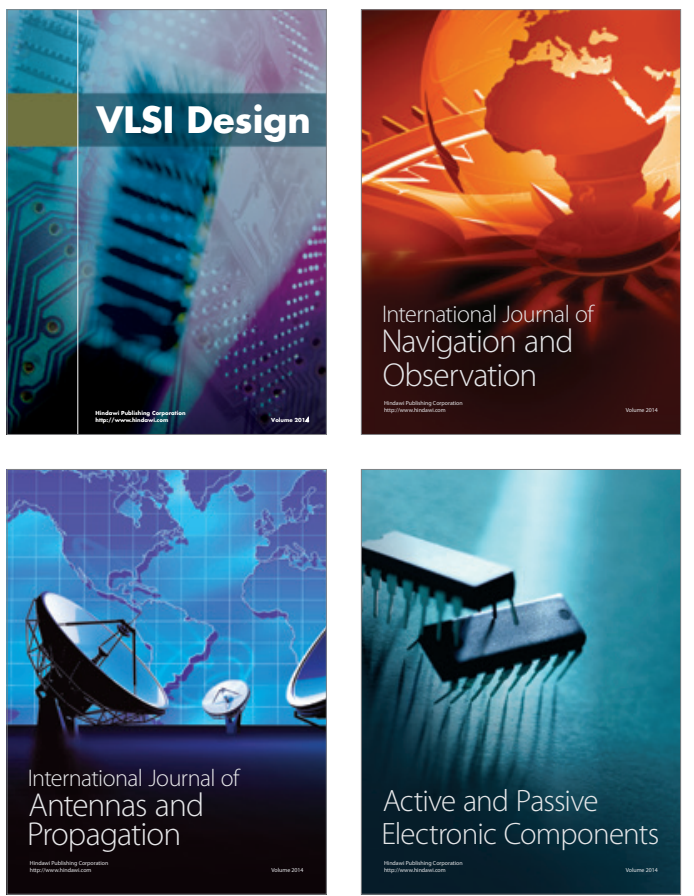
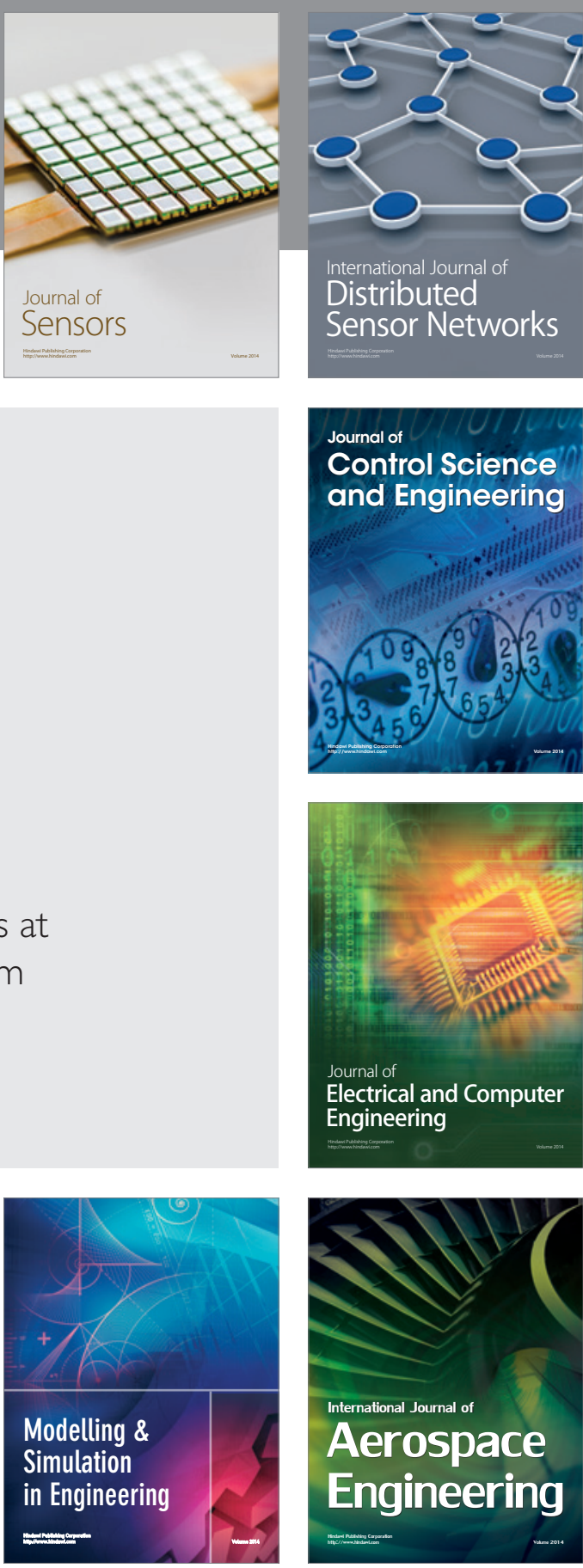

International Journal of

Distributed

Sensor Networks

Journal of

Control Science

and Engineering
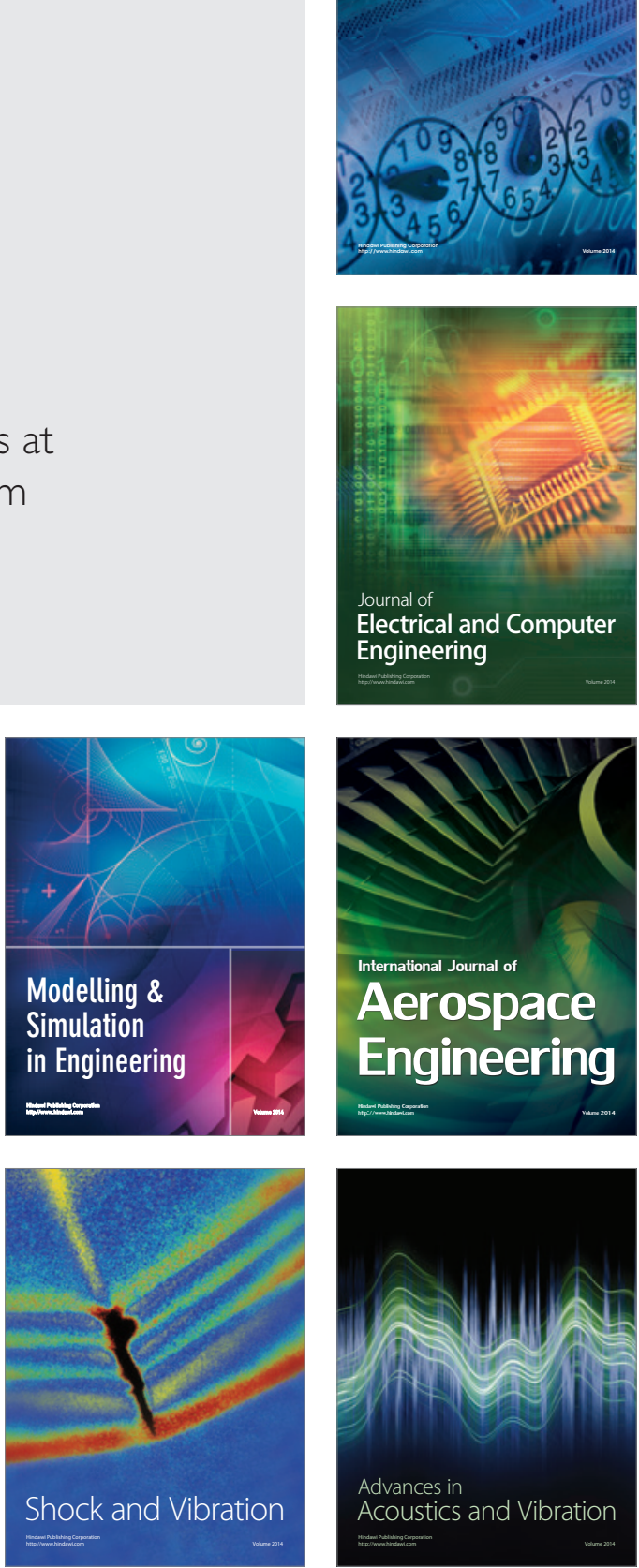